\title{
Study on the Preparation and PTC Behavior Of Graphene/ Polyvinylidene Fluoride Composites
}

\author{
HONG Liang $\mathrm{Hu}^{*}$, MING Yue Zhao and LI Guang Xiao \\ College of Materials Science and Engineering, Jilin jianzhu University, Changchun 130118 \\ honglianghu@126.com,xlg627@163.com
}

\begin{abstract}
Graphene/Polyvinylidene fluoride (PVDF) conductive composite materials were prepared by co-solvent method. GO were stably dispersed in $\mathrm{H}_{2} \mathrm{O} / \mathrm{N}, \mathrm{N}$-dimethylformamide (DMF) $(1: 9$, volume ratio) medium. After sonication, a clear stable brown solution was prepared without any visible aggregation.Changes in the internal structure and crystallization properties is demonstrated of the graphene/PVDF composites by scanning electron microscopy (SEM) and differential scanning calorimetry discussion(DSC).It was shown that in the 1:9 mixed solvent of $\mathrm{H}_{2} \mathrm{O} / \mathrm{DMF}$ can improve $\mathrm{GO}$ concentration which increased 2.98 times and can form a homogeneous stable solution. The resistance decreases with temperature, indicating an NTC behavior and the room-temperature resistivity downward trend during the thermal cycles
\end{abstract}

Keywords: Graphene, Co-solvent method, PVDF, Thermal reduction, PTC

\section{Introduction}

Polymer-based PTC materials are a kind of thermo-sensitive materials which are prepared with the polymer as the substrate and the addition of some conductive fillers such as carbon black, carbon fiber, and metal powder etc. However, graphene attracts widespread attention in the field of polymer due to the excellent electrical, mechanical, thermal properties compared to conventional polymer conductive filler ${ }^{[1-4]}$. But, due to the strong van der Waals force of graphene layers, the reunite and dispersed problems remain to be solved ofgraphene in the preparation process of polymer composites.

Recent studies have shown that RGO can have an ultrasonic exfoliation in polar solvents such as dimethyl formamide (DMF), N-methyl pyrrolidone (NMP) ${ }^{[5-6]}$. But, it is not suitable for the preparation of polymer nanocomposites due to the low concentration and can't steady state exist of RGO.So aiming at the above problems, we put forward using $\mathrm{H}_{2} \mathrm{O}$ and DMF mixed solvent,significantly improved the dispersion performance of RGO. At the same time RGO/PVDF composites were prepared by thermal reduction process and its PTC behavior was studied.

\footnotetext{
*Corresponding author: honglianghu@126.com
} 


\section{Experimental Procedures}

\subsection{Reagents and instruments}

Sodium nitrate, potassium permanganate, sulfuric acid, hydrogen peroxide, hydrochloric acid, nitric acid, N, N-dimethyl formamide were bought in Beijing Chemical Works, China. Deionized water was prepared by ourselves. Graphite was bought in Qing Dao ORR Graphite Co., LTD, China which purity is $99.9 \%$. Polyvinylidene fluoride (PVDF) FR903 was bought in Shang Hai $3 \mathrm{f}$ New Material Co.,Ltd, China.its density is $1.77 \mathrm{~g} / \mathrm{cm}^{3}$. Electric vacuum drying oven DZF-6050 was bought in Shang Hai Yi Heng Scientific Instrument Co., LTD, China. Digital multimeter VC9808 was bought in Shen Zhen Victory High Electronic Technology Co.,LTD, China. Electric mixer H20 was bought in Shang Hai Heng Chuan Machinery Equipment Co.,LTD, China.Transmission electron microscopy (TEM) JEM-1400 was bought in Shang Hai Bai He Instrument Technology Co., LTD, China. Digital thermometer TM-902C was bought in Shen Zhen Jing Teng Wei Industry Co., LTD, China. Plate vulcanizing machine was bought in Ying Kou Emerging Trial Machinery Works, China.Scanning electron microscope (SEM) SSX-550 was bought in Shen Zhen Rui Sheng Technology Co., LTD, China. Atomic force microscope (AFM) SPA-400 was bought in Juli Technology Co., LTD, China.

\subsection{Experimental}

GO was prepared from natural graphite powder according to Hummers' method ${ }^{[7]}$, GO sheets were dispersed in $\mathrm{H}_{2} \mathrm{O} / \mathrm{DMF}$ mixed solvent $\left(\mathrm{H}_{2} \mathrm{O} / \mathrm{DMF}=1: 9, \mathrm{~V} / \mathrm{V}\right)$ solution by sonication. Then the mica sheet which spined this solution was observed by AFM. GO was in ultrasonic treatment for two hours in $\mathrm{H}_{2} \mathrm{O}$ /DMF mixed solvent, subsequently PVDF was added to this mixed solvent, and we stirred this mixed solvent at $85^{\circ} \mathrm{C}$ for $1 \mathrm{~h}$ and dried this solution. After the composite material was hot pressed at $200^{\circ} \mathrm{C}$ for $2 \mathrm{~h}$.

\section{Results and Discussions}

\subsection{Preparation and characterization of composite materials}

After ultrasonic treatment for $\mathrm{GO}$ in a ratio of 1:9 $\mathrm{H}_{2} \mathrm{O} / \mathrm{DMF}$ in the mixed solvent, we can obtain uniform and stable yellow solution (Fig.1a). Through the AFM analysis of GO, we found that the thickness of the GO film is $0.814 \mathrm{~nm}$ less than $1 \mathrm{~nm}$ (Fig.1b), which indicates that the GO can be fully stripped in the mixed solvent of $\mathrm{H}_{2} \mathrm{O} / \mathrm{DMF}$. In the same time, by researching the GO dispersion properties of different solvent systems, we found that in the mixed solvent system of $\mathrm{H}_{2} \mathrm{O} / \mathrm{DMF}$ the introduction of $\mathrm{H}_{2} \mathrm{O}$ improves the dispersion ability(Fig.1c) .The concentration of $\mathrm{GO}$ in the mixed solvent of $\mathrm{H}_{2} \mathrm{O} / \mathrm{DMF}$ is $1.94 \mathrm{mg} / \mathrm{ml}$, which increases by 2.98 times compared with the concentration which is 0.65 $\mathrm{mg} / \mathrm{ml}$ in the single solvent of DMF. The analysis shows that the phenomenon is mainly attributed to the $\mathrm{H}_{2} \mathrm{O}$ molecules are more prone to penetrate the GO film than the DMF molecules. The $\mathrm{H}_{2} \mathrm{O}$ molecules can destroy the hydrogen bonds between the lamellae,thus increasing the concentration of $\mathrm{GO}^{[8]}$. 

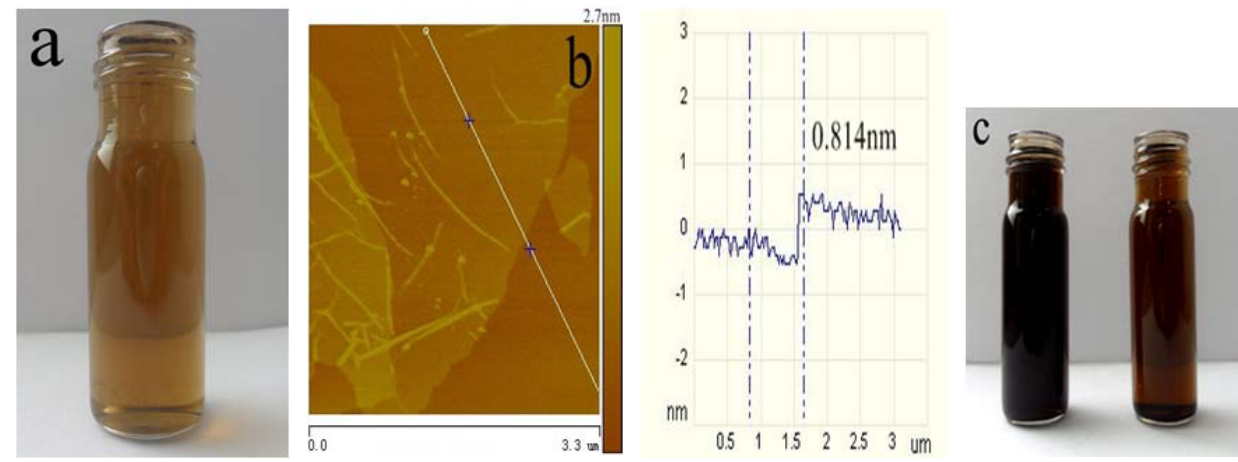

Fig. 1 (a) GO solution(H2O/DMF=1:9 volume ration),(b) AFM image of exfoliated GO sheets on a

mica substrate with height profile from the solution in (a),(c) From left to right :photos of GO dispersed in different solvents: $\mathrm{H}_{2} \mathrm{O} / \mathrm{DMF}=1$ :9, DMF

To fully obtain the influence of the production condition on the inner structure of the composite, the SEM analysis of the composite RGO/PVDF was performed, as portrayed in Fig. 2. From the picture, due to the presence of the water molecular, the multiporous sol-gel structure of GO/PVDF composite was observed(Fig. 2a), which is similar to the result studied of Zha et al. However, under the condition of $200^{\circ} \mathrm{C}$ and $10 \mathrm{Mpa}$, the polymer would go through the fusion process and subsequently the inner porous structure of the fused material will contract and finally disappear as illustrated in Fig. 2b, elimination the effect of the porous structure on the later electrical properties of the composites, while during the same process, GO was reduced under the high temperature. Ultimately, the conductive composite RGO/PVDF was prepared by thermal reduction process.
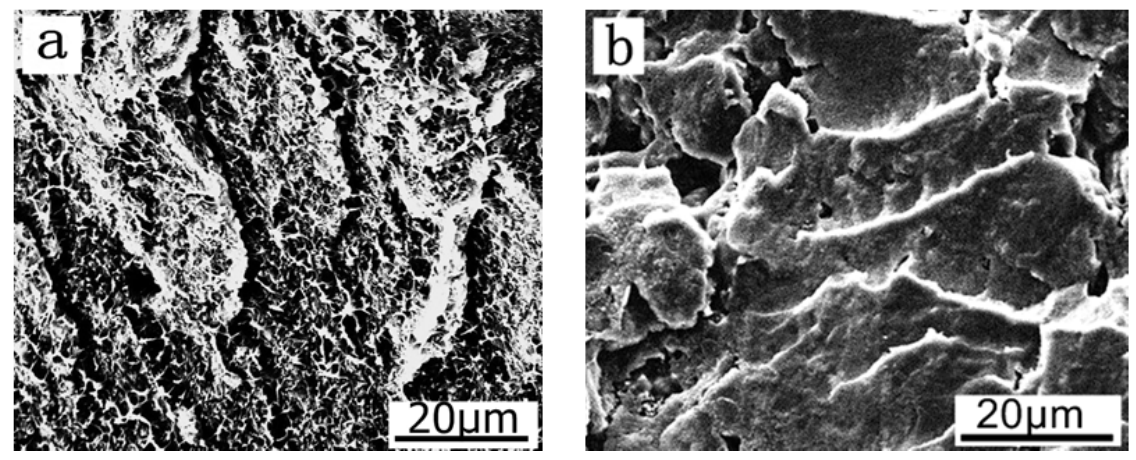

Fig.2 The SEM image of fracture surface of GO/PVDF and RGO/PVDF composites. 


\subsection{The PTC/NTC effect of the composite RGO/PVDF}

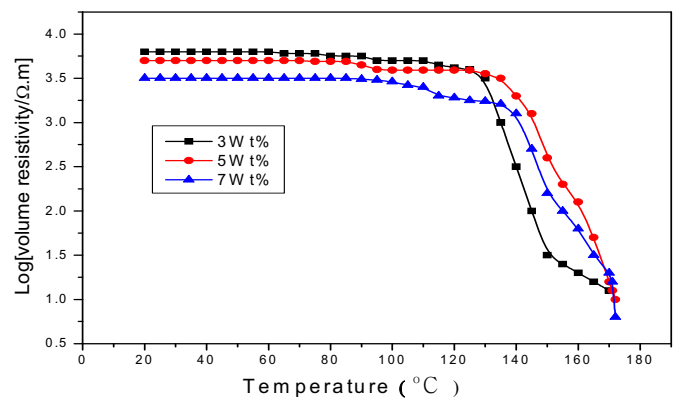

Fig.3 Resistivity-temperature curves of RGO/PVDF composites with different RGO weight percents

Wang Ke and other peoples had a research and think that when the temperature exceeds the polymer melting point, the flow property of polymer was enhanced and the viscosity was decreased, at the same time, the conductive particles also get more heat energy made them move more easily and strengthen the role of agglomeration. Causing a agglomeration of the filler particles in the polymer matrix, to reform a conductive path, so that the resistance decreased to produce NTC effect ${ }^{[9]}$. At the same time, from the past studies for the GP/PVDF, CF/PVDF, CB/PVDF conductive composite we found that all the composite materials show the PTC effect and the NTC transition temperature of the composite materials appears after the polymer matrix melting point. But this experimental results showed that the NTC transition temperature of RGO/PVDF conductive composite was lower than $160^{\circ} \mathrm{C}$ which made by co-solvent method, and with the increase of filler content it showed significant NTC effect (Fig.3).we think that this phenomenon due to the RGO had a larger surface area, which leaded to its contact resistance make major contributions, and the expansion effect of the polymer with the increase of temperature can not destroy the conductive network formed by $\mathrm{RGO}^{[10]}$. Therefore, when the temperature reaches the vicinity of the melting point of the polymer, the matrix liquidity increased, RGO fillers are more likely to migrate and agglomerate, thecapability to reform new conductive network was enhanced, making the volume resistance of the composite fell to three orders of magnitude, rendering the NTC effect significantly.

\subsection{The thermostability of the resistance of the composite RGO/PVDF}

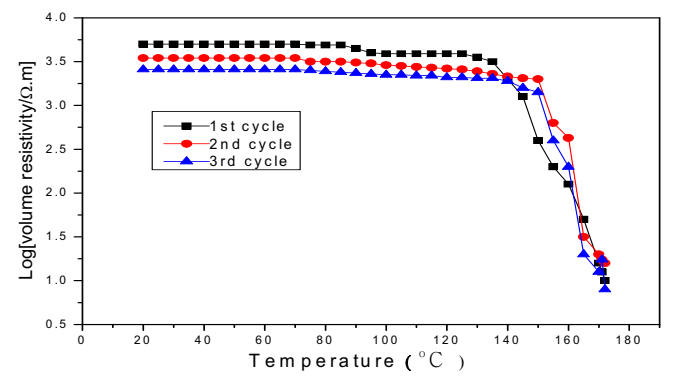

Fig.4 Resistivity-temperature behaviour of the $5 \mathrm{wt} \%$ RGO-filled PVDF composite with various numbers of thermal cycles 
The study shows that the distribution of the fillers before the test was frozen. But the distribution of the fillers was dynamic through heating. With the increase of temperature, the fusion of the crystal zone occurred, the viscosity of the system decreased and the fillers diffused to the amorphous region, after the thermal cycling, fillers tend to be uniformlydistributed and the ability to form a conductive path reduced. Thus the room temperature resistance of the composite is elevated ${ }^{[11]}$.In contrast, this experiment shows that, the room temperature resistance of the RGO/PVDF conductive composite material showed a declinetrend after the cycling test, as shown in Fig.4.In order to further expound the cause of the room temperature resistance declined after cycling, the experimental have a DSC test to RGO conductive composites which content is $5 \mathrm{wt} \%$,as shown in Fig.5.

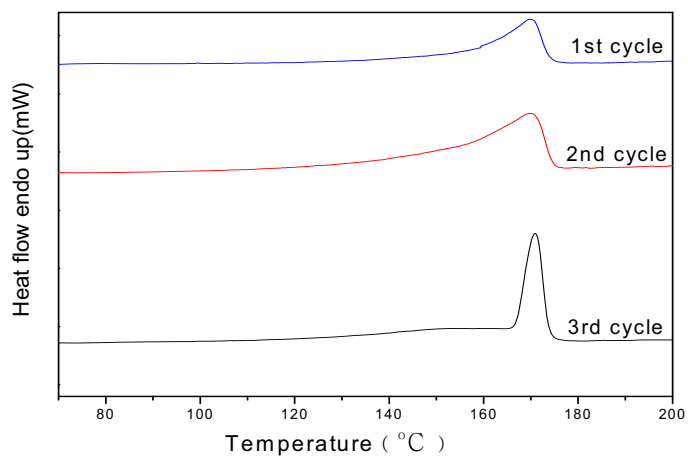

Fig.5. DSC curves of $5 \mathrm{wt} \% \mathrm{RGO} / \mathrm{PVDF}$ composites with three thermal cycles

The DSC test of the RGO/PVDF conductive composites was carried out after the thermal cycling test and found that RGO / PVDF composites corresponding peak melting temperatures were $170.17^{\circ} \mathrm{C}, 170.0^{\circ} \mathrm{C}$ and $170.83^{\circ} \mathrm{C}$, as shown in Fig.5.Through calculating the formula of composite crystallinity and we can see that the crystallinity of RGO/PVDF composites after three cycles was $43.78 \%, 44.89 \%$ and $45.29 \%$. The experimental results show that the peak melting temperatures of the RGO/PVDF composites has not changed significantly after thermal cycling and still near $170^{\circ} \mathrm{C}$. However, the crystallinity of the compositematerial after thermal cycling test showed an upward trend. Therefore, we believe that the decrease of RGO/PVDF composite temperature resistance in the process of cyclic testing was due to the increase of the internal crystallinity of the material. Prompting the amorphous zone concentration of RGO fillers increased and the room temperature resistance decreased.

$$
\begin{gathered}
\text { Crystallinitye }(\%)=(\Delta \mathrm{Hm} / \Delta \mathrm{H} 100) \times 100 \\
\Delta \mathrm{H} 100=104.7 \mathrm{~J} / \mathrm{g}
\end{gathered}
$$

Apart from enhancing the degree of crystallinity is the cause of RGO / PVDF composite material room temperature resistance declined. we believe that the possible reasons for the decrease of the room temperature resistance of RGO/PVDF composites are as follows. One hand, because of RGO particles is a large specific surface area, every layers connect closely and contact resistance makes major contributions. The ability of forming the conductive network to resist damage during the melt of the polymer matrix was improved. On the other hand, we speculate that the lamellar structure of the RGO is a flexible particle compared with the $\mathrm{CB}$ and $\mathrm{CF}$ when the material expanded. RGO particles had a rearrangement or deformation rather than the destruction of the conductive network, and eventually lead to the room temperature resistance of the RGO/PVDF conductive composite decreased after the cycling test. 


\section{Conclusion}

The experimental results show that GO can form stable homogeneous solution in $\mathrm{H}_{2} \mathrm{O} / \mathrm{DMF}$ mixed solvent, the thickness of GO layer was less than $1 \mathrm{~nm}$ and good conductive network is formed after hot reduction. The concentration of GO in the mixed solvent system was 2.98 times compared with the single solvent of DMF. RGO/PVDF composites showed NTC effect. As the thermal cycling, the room temperature resistance of RGO/PVDF composites showed a downward trend due to the improvement of crystallinity.

\section{References}

1. Stankovich S, Dikin DA, Dommett GHB, Kohlhaas KM, Zimney EJ, Stach EA, et al. Graphene-based composite materials[J].Nature, 2006, 442(7100): 282-286.

2. Steurer P, Wissert R, Thomann R, Muelhaupt R. Functionalized graphenes and thermoplastic nanocomposites based upon expanded graphite oxide[J].Macromol Rapid Commun, 2009, 30: 316-327.

3. Kuila T, Bose S, Hong CE, Uddin ME, Khanra P, Kim NH, et al. Preparation of Functionalized Gra- phene/Linear Low Density Polyethylene Composites by a Solution Mixing Method[J]. Carbon, 2011, 49(3): 1033-1037.

4. Spitalsky Z, Tasis D, Papagelis K, Galiotis C. Carbon nanotube-polymer composites: chemistry, processing, mechanical and electrical properties[J].Prog Polym Sci, 2010, 35(30): 357-401.

5. Yu Jh, Jiang $\mathrm{Pk}, \mathrm{Wu} \mathrm{C}, \mathrm{W} \mathrm{L}, \mathrm{Wu} \mathrm{X}$ f. Graphene nanocomposites based on poly(vinylidene fluoride):structure and properties[J].Polym. Compos, 2011, 32(10): 1483-1491.

6. Pham V H, Cuong T V, Hur S H, Oh E, Kim E J, Shin E W, Chung J S. J. Chemical functionalization of graphene sheets by solvothermal reduction of a graphene oxide suspension in N-methyl-2-pyrrolidone [J].Mater. Chem., 2011, 21(10): 3371-3377.

7. Hummers WS, Offeman RE. Preparation of graphitic oxide[J]. J Am Chem Soc, 1958, 80(6): 1339.

8. Zha D A, Mei SL, Wang ZY, Li HJ , Shi ZJ, Jin ZX. Superhydrophobic polyvinylidene fluoride/graphene porous materials[J]. Carbon, 2011, 49(15): 5166-5172.

9. DI W H, ZHANG G, XU J N, PENG Y, WANG X J, XIE Z Y. J. Positive temperature coefficient/negative temperature coefficient effect of low density polyethylene filled with a mixture of carbon black and carbon fiber[J]. Polym. Sci., Part B: Polym. Phys, 2003, 41(23): 3094-3101.

10. ANSARI S, GIANNELIS E P. J. Functionalized graphene sheet-poly (vinylidene fluoride) conductive nanocomposites[J]. Polym. Sci., Part B: Polym. Phys, 2009, 47(9): 888-897.

11. ZHANG C, MA C A,WANG P, et al. Temperature dependence of electrical resistivity for carbon black filled ultra-high molecular weight polyethylene composites prepared by hot compaction[J].Carbon,2005,43:2544-2553. 